\title{
EFEITO DE ALGUMAS VARIÁVEIS DE PROCESSAMENTO NAS PROPRIEDADES FÍSICAS DE PAINÉIS DE MADEIRA DE PARTÍCULAS ALONGADAS
}

\section{EFFECT OF SOME VARIABLES OF PROCESSING IN THE PHYSICAL PROPERTIES OF STRANDBOARD WOOD PANELS}

\author{
Graciane Angélica da Silva ${ }^{1}$ Lourival Marin Mendes ${ }^{2}$ Paulo Fernando Trugilho ${ }^{2}$ \\ Fábio Akira Mori ${ }^{2}$ Izaías Fernandes dos Santos ${ }^{3}$ Franciane Andrade de Pádua ${ }^{4}$ \\ RESUMO
}

A produção de painéis envolve parâmetros de processamento que interferem nas propriedades e na umidade de equilíbrio dos painéis. Dentre os parâmetros do processamento, podemos citar a massa específica dos painéis, razão de compactação, tipo e conteúdo de adesivo, conteúdo de parafina, dimensão e umidade das partículas, tempo de fechamento da prensa, temperatura e tempo de prensagem e pressão específica. Todos esses parâmetros podem ser controlados, visando a melhorar as qualidades do painel. Este trabalho teve como objetivo estudar a influência das variáveis de processamento (teor de resina, temperatura de prensagem e tempo de prensagem) nas propriedades físicas dos painéis. No Laboratório de Tecnologia e Produtos Florestais da Universidade Federal do Paraná, foram produzidos 27 painéis homogêneos de camada única sem orientação com partículas strand, com as dimensões de 480 × 480 × 15 mm; e no Laboratório de Tecnologia da Madeira da Universidade Federal de Lavras, foram gerados os corpos-de-prova para estudo das propriedades físicas (inchamento em espessura, absorção de água e taxa de não-retorno - TNRE) dos painéis. Os resultados demonstraram que o teor de resina, a temperatura e o tempo de prensagem afetam, de forma significativa, a retenção de água pelos painéis.

Palavras-chave: propriedades físicas; variáveis de processamento; painéis de madeira, aglomerados estruturais.

\begin{abstract}
The production of panels encompasses processing parameters that interfere in the properties and in the equilibrium moisture content of the panels such as specific gravities, compaction ratio, type and amount of adhesive, paraffin content, dimension and moisture content of the particles, time of closing the press, temperature and time of pressing and specific pressure. All these parameters can be controlled aiming to improve the quality of the panels. The study determined the influence of some processing variables (resin content, temperature and time of pressing) in the physical properties of the panels. At the Wood Technology and Forestry Products Laboratory of Universidade Federal do Paraná, 27 homogeneous single layer measuring $480 \times 480 \times 15 \mathrm{~mm}$ were produced with strands without orientation and, at the Wood Technology and Forestry Products Laboratory of Universidade Federal Lavras, samples for the physical properties testing (thickness swelling, water absorption and the nonrecoverable tax - TNRE) were generated. Results demonstrated that the resin content, temperature and time of pressing affect drastically the retention of water by the panels.
\end{abstract}

Key words: physical properties; processing variables; wood-based panel; strandboards.

\section{INTRODUÇÃO}

Quase todos os parâmetros, mostrados na Tabela 1 interagem entre si, nos dois sentidos, na produção de painéis particulados de madeira (Maloney, 1993). Por conseguinte, a mudança de um desses fatores resultará na alteração de vários outros relacionados com o processo de formação do painel, ou seja, um parâmetro não pode ser considerado isoladamente, como se pudesse ter manipulação independente e fácil, a

1. Engenheira Florestal e Mestre em Ciência e Tecnologia da Madeira pela Universidade Federal de Lavras, Rua Nelo Totti, 183, Centro, CEP: 37405-000, Monsenhor Paulo (MG). gracianeas@yahoo.com.br

2. Engenheiro Florestal, Dr., Professor Adjunto do Departamento de Ciências Florestais, Universidade Federal de Lavras, Caixa Postal 37, CEP: 37200-000, Lavras (MG). lourival@ufla.br/trugilho@ufla.br/morif@ufla.br

3. Acadêmico do Curso de Graduação em Engenharia Florestal, Universidade Federal de Lavras, Caixa Postal 37 , CEP: 37200-000, Lavras (MG). ifsantos@eflorestal.ufla.br

4. Engenheira Florestal, Doutoranda em Ciência e Tecnologia da Madeira, Universidade Federal de Lavras, Rua Deputado Castejon Branco, 110, Jardim Fabiana, CEP: 37200-000, Lavras (MG). franpadua@zipmail.com.br

Recebido para publicação em 14/05/2004 e aceito em 17/02/2006. 
fim de controlar o processo de formação do painel de forma adequada. Contudo, uma vez aceita a existência dessa inter-relação entre certo número de parâmetros, um alcance mais completo do processo pode ser atingido, e a manipulação real pode ser desenvolvida com sucesso para o controle da maior parte do processo.

TABELA 1: Fatores que afetam as propriedades dos painéis particulados.

TABLE 1: Factors that affect the properties of the wood-based panels.

\begin{tabular}{ll}
\hline Inerentes a Madeira & Inerentes ao Processo \\
\hline Espécies & Massa específica dos painéis \\
Massa específica da madeira & Razão de compactação \\
pH & Composição dos painéis \\
Teor de umidade & Resina e parafina \\
Extrativos & Dimensão e orientação das partículas \\
Local de crescimento & Umidade das partículas \\
Idade cambial & Tempo de fechamento da prensa e de prensagem \\
Substâncias estranhas & Temperatura de prensagem \\
Forma do Tronco & Pressão específica \\
\hline
\end{tabular}

Fonte: Mendes e Iwakiri (2002).

Dentre os parâmetros de processamento dos painéis que podem ser controlados visando a melhorar a qualidade e minimizar o custo, pode-se destacar: o teor de resina e tempo, e temperatura de prensagem os quais são mencionados a seguir.

\section{Resina}

$\mathrm{Na}$ produção de chapas de partículas de madeira, o adesivo é o componente de maior custo de produção, portanto, a quantidade a ser aplicada deve ser otimizada, em função das propriedades requeridas para a finalidade de uso que se destina.

Segundo Cloutier (1998), os quatro principais tipos de resinas, atualmente empregadas na indústria de painéis compostos à base de madeira, são os seguintes: uréia-formaldeído (UF), melamina-formaldeído (MF), fenol-formaldeído (FF) e difenil metano di-isocianato (MDI).

\section{Tempo de Prensagem}

O tempo de prensagem é o tempo decorrido entre o momento da consolidação do colchão de partículas em sua espessura até o momento de abertura dos pratos da prensa, podendo variar em torno de 6 a 12 minutos.

O tempo de prensagem deverá ser o suficiente para que o centro da chapa atinja a temperatura ideal para a cura da resina e que seja também suficiente para a migração da umidade pelas bordas da chapa.

Segundo Matos (1988), reduções no tempo de prensagem dos painéis são mais desejáveis, pois implica em uma maior produção por unidade de tempo, bem como provoca uma redução no consumo de energia. O mesmo autor relata que o principal efeito do decréscimo no tempo de prensagem é a redução nas propriedades de resistência.

\section{Temperatura de Prensagem}

A principal função da temperatura da prensa, na produção de painéis de partículas, é a aceleração da polimerização do adesivo distribuído entre as partículas e a plasticização da madeira (Matos, 1988).

\section{Propriedades Físicas dos Painéis}

\section{Absorção de Água e Inchamento em espessura}

Absorção de água é a expressão percentual da quantidade de água absorvida o que ocorre quando uma amostra acondicionada a $20^{\circ} \mathrm{C}$ e $65 \%$ de UR (umidade de equilíbrio em torno de $12 \%$ ) é imersa em água por 2 e 24 horas, de acordo com a Norma ASTM, XXX. Segundo Brito (1995), o inchamento em espessura é uma das propriedades mais importantes em termos de estabilidade dimensional dos painéis. O inchamento em espessura pode ser afetado pela espécie de madeira, geometria das partículas, massa específica dos painéis, nível de resina, nível de parafina, eficiência da aplicação da cola e condições de prensagem. 


\section{Taxa de não-retorno em espessura (TNRE)}

Segundo Iwakiri (1989), a exposição do painel a um ciclo de alta e baixa umidade relativa, em que ocorre primeiro o inchamento e depois a contração, resulta em um inchamento residual por causa da liberação das tensões de compressão impostas aos painéis, e é denominada de taxa de não-retorno em espessura. Esse efeito pode ser reduzido ou eliminado por meio de tratamentos especiais como pósvaporização e pós-aquecimento dos painéis, embora esses métodos tenham aplicações limitantes.

Em estudo realizado por Matos (1988), para painéis Waferboard produzidos com Pinus taeda em diferentes condições de prensagem, foram observadas uma forte influência da umidade das partículas e presença de parafina sobre o inchamento residual. $\mathrm{O}$ autor relata que, com a aplicação de $1 \%$ de parafina, $o$ inchamento residual é reduzido.

Este trabalho teve como finalidade estudar a influência de algumas variáveis de processamento (teor de resina, temperatura de prensagem e tempo de prensagem) nas propriedades físicas dos painéis, utilizando o teste de identidade de modelos.

\section{MATERIAIS E MÉTODOS}

Foram utilizados, para este estudo, madeira de Eucalyptus oriunda de testes clonais instalados pela Companhia Mineira de Metais (CMM-Agro), localizada entre os municípios de Paracatu e Vazante, região

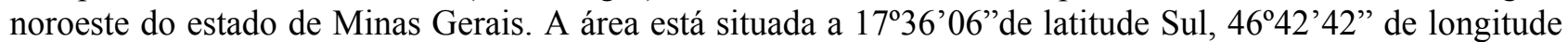
Oeste de Greenwich e a $550 \mathrm{~m}$ de altitude. Segundo classificação de Koppen, o clima é do tipo Aw, tropical úmido de savana, com inverno seco e verão chuvoso. A temperatura média anual é de $24^{\circ} \mathrm{C}$ e a precipitação média anual é de $1,450 \mathrm{~mm}$.

No Laboratório de Tecnologia da madeira da UFLA, foram geradas as partículas tipo strand. Já, no Laboratório de Painéis de Madeira do Departamento de Engenharia e Tecnologia Florestal da UFPR, foram produzidos 27 painéis homogêneos de camada única sem orientação com partículas strand, com as dimensões nominais de 480 × 480 × $15 \mathrm{~mm}$, e com massa específica de $0,65 \mathrm{~g} / \mathrm{cm}^{3}$. Na Tabela 2, observa-se o delineamento experimental utilizado para os tratamentos.

\section{Avaliação das propriedades físicas dos painéis}

No Laboratório de Tecnologia da Madeira da UFLA, os painéis foram cortados em corpos-de-prova com as dimensões de $150 \mathrm{~mm}$ de largura por $150 \mathrm{~mm}$ de comprimento para estudo de absorção de água (AA), inchamento em espessura (IE) e taxa de não-retorno em espessura (TNRE). De cada painel foram cortados quatro corpos-de-prova, os quais inicialmente foram pesados e medidos (largura, comprimento e espessura), em seguida foram submersos em água e após 2 e 24 horas foram feitas, novamente, medições da espessura e peso.

As propriedades físicas foram determinadas de acordo com as normas ASTM:

- Absorção de água (AA): ASTM D 1037-91 (1998).

- Inchamento em espessura (IE): norma ASTM D 1037-91 (1998).

Para a análise das propriedades físicas (água na forma líquida), adotou-se o delineamento inteiramente casualizado, com 27 tratamentos e quatro repetições. Os tratamentos constituíram um fatorial $3^{3}$, envolvendo, portanto, três fatores, cada um com três níveis (teor de resina de 3, 4,5 e 6\%; temperatura de prensagem de 160,180 e $200^{\circ} \mathrm{C}$; tempo de prensagem de 6,8 e $10 \mathrm{~min}$ ).

Os dados foram analisados, estatisticamente, por meio da metodologia de superfície de resposta, em que foi utilizado o modelo polinomial quadrático.

Foi realizado o ajuste de uma equação de regressão para cada variável dependente (inchamento 2 horas, inchamento 24 horas, absorção 2 horas, absorção 24 horas e TNRE), em cada teor de resina (3, 4,5 e $6 \%$ ), foram comparados pelo teste de identidade de modelos. 
TABELA 2: Delineamento experimental utilizado na produção dos painéis de madeira.

TABLE 2: Experimental delineation used in the production of the wood-based panels.

\begin{tabular}{c|c|c|c}
\hline Tratamento & TR $(\%)$ & $\mathrm{T}^{\circ} \mathrm{P}\left({ }^{\circ} \mathrm{C}\right)$ & $\mathrm{T}^{\prime} \mathrm{P}(\mathrm{min})$ \\
\hline 1 & 3 & 160 & 6 \\
2 & 3 & 160 & 8 \\
3 & 3 & 160 & 10 \\
4 & 3 & 180 & 6 \\
5 & 3 & 180 & 8 \\
6 & 3 & 180 & 10 \\
7 & 3 & 200 & 6 \\
8 & 3 & 200 & 8 \\
9 & 3 & 200 & 10 \\
10 & 4,5 & 160 & 6 \\
11 & 4,5 & 160 & 8 \\
12 & 4,5 & 160 & 10 \\
13 & 4,5 & 180 & 6 \\
14 & 4,5 & 180 & 8 \\
15 & 4,5 & 180 & 10 \\
16 & 4,5 & 200 & 6 \\
17 & 4,5 & 200 & 8 \\
18 & 4,5 & 200 & 10 \\
19 & 6 & 160 & 6 \\
20 & 6 & 160 & 8 \\
21 & 6 & 160 & 10 \\
22 & 6 & 180 & 6 \\
23 & 6 & 180 & 8 \\
24 & 6 & 180 & 10 \\
25 & 6 & 200 & 6 \\
26 & 6 & 200 & 80 \\
27 & 6 & $-T 0$ & \\
\hline
\end{tabular}

Em que: $\mathrm{TR}=$ Teor de resina; $\mathrm{T}^{0} \mathrm{P}=$ Temperatura de prensagem; $\mathrm{T}$ 'P =Tempo de prensagem.

O modelo estatístico utilizado foi:

$\mathrm{Y}=\beta_{0}+\beta_{1} \mathrm{X}_{1}+\beta_{2} \mathrm{X}_{2}+\beta_{11} \mathrm{X}_{1}^{2}+\beta_{22} \mathrm{X}_{2}^{2}+\beta_{12} \mathrm{X}_{1} \mathrm{X}_{2}+e$

Em que: $\mathrm{Y}=$ variável dependente; $\beta_{0}=$ constante de regressão; $\beta_{1}, \beta_{2}, \beta_{11}, \beta_{22}, \beta_{12}=$ coeficiente de regressão; $\mathrm{X}_{1}=$ temperatura de prensagem; $\mathrm{X}_{2}=$ tempo de prensagem; e = erro experimental, suposto normal e independente.

O modelo polinomial quadrático ajustado é:

$\mathrm{Y}=b_{0}+b_{1} \mathrm{X}_{1}+b_{2} \mathrm{X}_{2}+b_{11} \mathrm{X}_{2}^{2}+b_{22} \mathrm{X}_{2}^{2}+b_{12} \mathrm{X}_{1} \mathrm{X}_{2}$

Em que: $b_{0}, b_{1}, b_{11}, b_{22}$ e $b_{12}=$ estimativas dos parâmetros $\beta_{0}, \beta_{1}, \beta_{2}, \beta_{11}, \beta_{22}, \beta_{12}$ respectivamente.

Com as equações das superfícies ajustadas para cada teor de resina, foram determinados os pontos críticos (pontos estacionários) e sua natureza, isto é, se o ponto foi de máximo, mínimo, sela ou nenhum dos três.

Para cada equação ajustada em cada teor de resina, obteve-se o ponto estacionário $\mathrm{S}$, bastando, para isto, tomar $\mathrm{Y}=0$ (diferencial de $\mathrm{Y}$ identidade nula) o que equivale a resolver o seguinte sistema: 


$$
\begin{aligned}
& \frac{\partial \mathrm{Y}}{\partial \mathrm{X}_{1}}=0 \quad \text { ou seja, } \quad \begin{array}{l}
2 b_{11} \mathrm{X}_{1}+b_{12} \mathrm{X}_{2}=-b_{1} \\
\frac{\partial \mathrm{Y}}{\partial \mathrm{X}_{2}}=0
\end{array} \quad b_{12} \mathrm{X}_{1}+2 b_{22} \mathrm{X}_{2}=-b_{2}
\end{aligned}
$$

Uma alternativa para estudar a natureza do ponto estacionário é por meio da classificação da forma quadrática.

$\frac{1}{2} \partial 2 \mathrm{Y}=\left[\begin{array}{ll}\partial \mathrm{X}_{1} & \partial \mathrm{X}_{2}\end{array}\right] \quad \mathrm{B}\left[\begin{array}{l}\partial \mathrm{X}_{1} \\ \partial \mathrm{X}_{2}\end{array}\right]$, obtendo-se os autovalores da matriz $\mathrm{B}$, a seguir:

$\mathrm{B}=\left[\begin{array}{cc}b_{11} & \frac{1}{2} b_{12} \\ \frac{1}{2} b_{12} & b_{22}\end{array}\right]$

Os autovalores são a solução da equação característica $|\mathrm{B}-\lambda I|=0$ isto é,

$\left[\begin{array}{cc}b_{11}-\lambda & \frac{1}{2} b_{12} \\ \frac{1}{2} b_{12} & b_{22}-\lambda\end{array}\right]=0$ as raízes da equação, m que: $b=b_{11}+b_{22} ;$ e $c=b_{11} b_{12}-\frac{1}{4} b_{12}{ }^{2}$

O sinal e a magnitude dos $\lambda$ 's são muito importantes para determinar a natureza do ponto estacionário. Se os $\lambda$ 's são todos positivos, tem-se um ponto de mínimo; se todos são negativos, tem-se um ponto de máximo; e se os sinais não forem iguais, tem-se um ponto de sela. A magnitude dos $\lambda$ 's pode oferecer muita informação a respeito do sistema. No caso de um dos $\lambda$ 's ser zero, tem-se uma aresta estacionária. A condição de aresta estacionária apresenta-se como um caso que limita a condição de ponto de máximo, mínimo ou sela. Na prática, é pouco provável que se tenha uma condição exata de aresta estacionária. Entretanto, se o valor de um dos $\lambda$ 's for bem pequeno, então o sistema aproximou-se de uma aresta estacionária. Se o ponto estacionário é um máximo e um dos $\lambda$ 's é quase zero, então, para todo propósito prático, o máximo não é unicamente esse ponto estacionário. Se o valor de $\lambda_{1}$ for positivo, $\lambda_{2}$ próximo de zero e o ponto estacionário fora da região experimental, essa condição se aproxima de uma queda na aresta; se $\lambda_{1}$ for negativo, tem-se uma subida na aresta (Meyrs, 1971).

As equações das superfícies ajustadas também foram avaliadas graficamente por meio de cortes na sua estrutura.

\section{RESULTADOS E DISCUSSÃO}

Na Tabela 3, estão apresentadas as equações das superfícies ajustadas para cada variável dependente e o seu respectivo coeficiente de determinação. Segundo Maloney (1993), todas as variáveis de produção interagem entre si, mas como o teor de resina representa o maior custo variável na manufatura de painéis particulados, optou-se pelo ajuste de equações, para cada nível de resina e realização do teste de identidade de modelos lineares.

Pode-se verificar que os coeficientes de regressão foram significativas para os níveis de resina e também nas equações comuns. Pela análise de variância e teste de identidade de modelos, para os três teores de resina $(3,4,5$ e $6 \%)$, isto é, a hipótese de igualdade das equações de regressão foi rejeitada a $5 \%$ de probabilidade, pelo teste $\mathrm{F}$, para inchamento em espessura, absorção de água e TNRE (Tabela 4).

Este fato indica que tais equações diferem estatisticamente $(p<0,05)$, não sendo possível substituí-las por uma equação comum (geral), o que evidencia, assim, a influência do teor de resina sobre as variáveis avaliadas no processo. Dessa forma, procurou-se fazer a avaliação das superfícies individuais por meio de cortes na sua estrutura.

De modo geral, houve uma tendência de redução nos valores médios de inchamento em espessura tanto para 2 como para 24 horas, quando o teor de resina foi aumentando de 3, 4,5 até $6 \%$. Essa tendência também foi observada por Mendes (2001), utilizando teores de resina de 4 e $6 \%$. 
TABELA 3: Equações das superfícies ajustadas com os seus respectivos coeficientes de determinação $\left(\mathrm{R}^{2}\right)$.

TABLE 3: Equations of the surfaces adjusted according to their respective coefficients of determination $\left(\mathrm{R}^{2}\right)$.

\begin{tabular}{|c|c|c|}
\hline \multicolumn{3}{|c|}{ Equações para inchamento 2 horas } \\
\hline Resina (\%) & & $\mathrm{R}^{2}$ \\
\hline 3 & $Y=634,8310-6,1703 X_{1}+5,4203 X_{2}+0,0126 X_{1}^{2}-1,9310 X_{2}^{2}+0,1307 X_{1} X_{2}$ & $0,855^{* *}$ \\
\hline 4,5 & $Y=198,2660-0,4450 X_{1}-26,0996 X_{2}-0,0013 X_{1}^{2}+0,7852 X_{2}^{2}+0,0764 X_{1} X_{2}$ & $0,812 * *$ \\
\hline 6 & $Y=115,7590+0,0887 X_{1}-23,9829 X_{2}-0,0013 X_{1}^{2}+0,8462 X_{2}^{2}+0,0466 X_{1} X_{2}$ & $0,789 * *$ \\
\hline Comum & $Y=316,6330-2,1760 X_{1}-14,8884 X_{2}+0,0033 X_{1}^{2}-0,0998 X_{2}^{2}+0,0846 X_{1} X_{2}$ & $0,253 * *$ \\
\hline \multicolumn{3}{|c|}{ Equações para inchamento 24 horas } \\
\hline 3 & $Y=664,8210-6,3541 X_{1}+4,7823 X_{2}+0,0130 X_{1}^{2}-1,8185 X_{2}^{2}+0,1266 X_{1} X_{2}$ & $0,838^{* *}$ \\
\hline 4,5 & $Y=337,8780-1,6663 X_{1}-32,6120 X_{2}+0,0017 X_{1}^{2}+1,0292 X_{2}^{2}+0,0924 X_{1} X_{2}$ & $0,866^{* *}$ \\
\hline 6 & $Y=266,2690-0,9691 X_{1}-35,9476 X_{2}+0,0010 X_{1}^{2}+1,2706 X_{2}^{2}+0,0727 X_{1} X_{2}$ & $0,863 * *$ \\
\hline Comum & $Y=423,0500-2,9971 X_{1}-21,2603 X_{2}+0,0052 X_{1}^{2}+0,1604 X_{2}^{2}+0,0973 X_{1} X_{2}$ & $0,265^{* *}$ \\
\hline \multicolumn{3}{|c|}{ Equações para absorção 2 horas } \\
\hline 3 & $\mathrm{Y}=721,6470-6,1362 \mathrm{X}_{1}-9,6070 \mathrm{X}_{2}+0,0119 \mathrm{X}_{1}^{2}-1,5423 \mathrm{X}_{2}^{2}+0,1717 \mathrm{X}_{1} \mathrm{X}_{2}$ & $0,862 * *$ \\
\hline 4,5 & $Y=-247,3080+4,2534 X_{1}-6,0767 X_{2}-0,0157 X_{1}^{2}-0,8787 X_{2}^{2}+0,1152 X_{1} X_{2}$ & $0,934 * *$ \\
\hline 6 & $Y=-829,4520+11,2692 X_{1}-34,3441 X_{2}-0,0307 X_{1}^{2}+2,2352 X_{2}^{2}-0,0157 X_{1} X_{2}$ & $0,595^{* *}$ \\
\hline Comum & $Y=-118,4420+3,1296 X_{1}-16,6767 X_{2}-0,0115 X_{1}^{2}+0,0619 X_{2}^{2}+0,0904 X_{1} X_{2}$ & $0,176^{* *}$ \\
\hline \multicolumn{3}{|c|}{ Equações para absorção 24 horas } \\
\hline 3 & $\mathrm{Y}=825,3910-6,1754 \mathrm{X}_{1}-33,6256 \mathrm{X}_{2}+0,0119 \mathrm{X}_{1}^{2}-0,0701 \mathrm{X}_{2}^{2}+0,1858 \mathrm{X}_{1} \mathrm{X}_{2}$ & $0,845^{* *}$ \\
\hline 4,5 & $Y=682,2010-4,7826 X_{1}-33,0969 X_{2}+0,0082 X_{1}^{2}-0,0059 X_{2}^{2}+0,1828 X_{1} X_{2}$ & $0,959 * *$ \\
\hline 6 & $Y=-252,4740+6,6925 X_{1}-65,9601 X_{2}-0,0209 X_{1}^{2}+2,9260 X_{2}^{2}+0,0907 X_{1} X_{2}$ & $0,644 * *$ \\
\hline Comum & $Y=418,3970-1,4221 X_{1}-44,2295 X_{2}-0,0003 X_{1}^{2}+0,9500 X_{2}^{2}+0,1531 X_{1} X_{2}$ & $0,214 * *$ \\
\hline \multicolumn{3}{|c|}{ Equações para TNRE } \\
\hline 3 & $Y=958,1250-9,2269 X_{1}-2,4198 X_{2}+0,0190 X_{1}^{2}-2,2997 X_{2}^{2}+0,2057 X_{1} X_{2}$ & $0,843 * *$ \\
\hline 4,5 & $Y=411,4390-2,7171 X_{1}-28,7873 X_{2}+0,0047 X_{1}^{2}+0,8571 X_{2}^{2}+0,0868 X_{1} X_{2}$ & $0,743 * *$ \\
\hline 6 & $Y=260,1140-1,0094 X_{1}-34,6600 X_{2}+0,0010 X_{1}^{2}+1,1765 X_{2}^{2}+0,0738 X_{1} X_{2}$ & $0,788 * *$ \\
\hline Comum & $Y=543,6100-4,3220 X_{1}-21,9576 X_{2}+0,0082 X_{1}^{2}-0,0908 X_{2}^{2}+0,1221 X_{1} X_{2}$ & $0,279 * *$ \\
\hline
\end{tabular}

Em que: $\mathrm{X}_{1}=$ Temperatura de prensagem; $\mathrm{X}_{2}=$ Tempo de prensagem; $* *=$ Significativo a $1 \%$ de probabilidade pelo teste $\mathrm{F}$.

O aumento da disponibilidade de resina, por área superficial de partículas, é a causa da diminuição da absorção de água, do inchamento em espessura e da TNRE. Quando o teor de resina aumentou de 3, 4,5 até $6 \%$, não só pela barreira física que é maior na linha de cola, mas também pela ocupação dos sítios higroscópicos da madeira $(\mathrm{OH})$, deixando o colchão menos reativo à água.

TABELA 4: Resumo da Análise de variância com o teste de identidade de modelos para as três porcentagens de resina, para inchamento em espessura (IE), absorção de água (AA) e taxa de não-retorno em espessura (TNRE).

TABLE 4: Summary of the analysis of variance with the test of identity of models for the three resin levels, thickness swelling (IE), water absorption (AA) and spring black (TNRE).

\begin{tabular}{l|l|c|c|c|c|c}
\hline \multirow{2}{*}{ FV } & GL & \multicolumn{5}{c}{ QM } \\
\cline { 2 - 7 } & & IE 2 horas & IE 24 horas & AA 2 horas & AA 24 horas & TNRE \\
\hline Parâmetros & 18 & 5811,345 & 8226,984 & 24078,34 & 42905,09 & 6004,923 \\
Redução (B) & 6 & 15798,79 & 22821,1 & 69438,04 & 125813,9 & 15710,84 \\
Redução (H0) & 12 & $817,6238^{*}$ & $929,9278^{*}$ & $1398,488^{*}$ & $1450,67^{*}$ & $1151,963^{*}$ \\
Resíduo & 90 & 21,45277 & 25,45031 & 93,43688 & 55,21129 & 25,11186 \\
\hline Total & 108 &
\end{tabular}

Em que: * = Significativo a 5\% de probabilidade, pelo teste $\mathrm{F}$.

As Figuras 1 e 2 ilustram as tendências de comportamento do inchamento em espessura 2 e 24 horas, em função dos teores de resina respectivamente. De acordo com essas figuras, verifica-se a existência de mesma tendência, para os inchamentos em espessura, 2 e 24 horas, em que se observa que, nos teores de resina de $3 \%$ e $4,5 \%$, à medida que aumenta a temperatura de prensagem, ocorre à diminuição do 
inchamento, e que o menor inchamento ocorreu na temperatura de $200^{\circ} \mathrm{C}$ e tempo de $6 \mathrm{~min}$.

Para o teor de resina de $6 \%$, à medida que aumenta a temperatura de prensagem o inchamento se mantém constante, sendo que o menor inchamento ocorreu na temperatura de $160^{\circ} \mathrm{C}$ e tempo de $10 \mathrm{~min}$.

Por apresentar tendência muito semelhante, pode-se realizar somente a avaliação de inchamento em espessura de 2 horas e, assim, poder reduzir o tempo e aumentar o número de avaliações dos painéis.

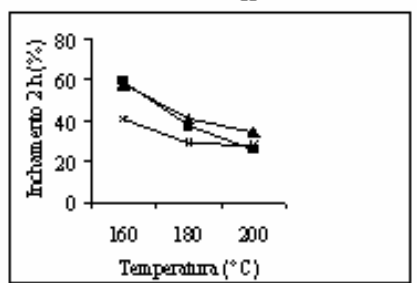

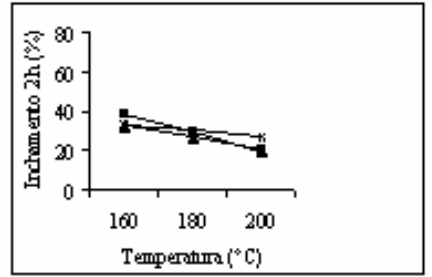

c
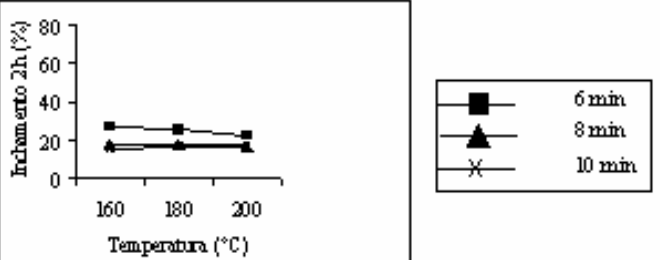

FIGURA 1: Cortes nas superfícies de resposta ajustada para a variável dependente inchamento, em espessura $2 \mathrm{~h}$, para os teores de resina 3\% (A), 4,5\% (B) e $6 \%$ (C).

FIGURE 1: Cuts in the surfaces of reply adjusted for the dependent variable thickness swelling $2 \mathrm{~h}$, based on resin contents of $3 \%(\mathrm{~A}), 4,5 \%(\mathrm{~B})$ and $6 \%(\mathrm{C})$.

A

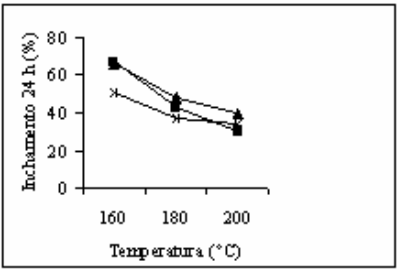

B

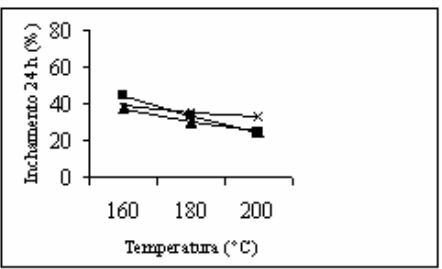

C
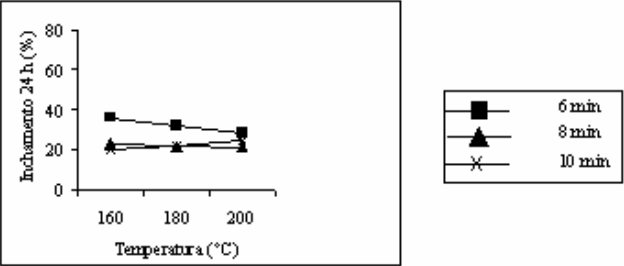

FIGURA 2: Cortes nas superfícies de resposta ajustada para a variável dependente inchamento, em espessura $24 \mathrm{~h}$, para os teores de resina $3 \%$ (A), $4,5 \%$ (B) e $6 \%$ (C).

FIGURE 2: Cuts in the surfaces of adjusted reply for the dependent variable thickness swelling $24 \mathrm{~h}$, based on resin contents of $3 \%(\mathrm{~A}), 4,5 \%(\mathrm{~B})$ and $6 \%(\mathrm{C})$.

As Figuras 3 e 4 ilustram as tendências de comportamento da absorção de água 2 e 24 horas, em função dos teores de resina respectivamente. Observando essas figuras, verifica-se também que existe semelhança de comportamento, ou tendência, para a absorção de água 2 e 24 horas. Para os teores de resina de 3 e 4,5\%, à medida que aumenta a temperatura de prensagem diminui a absorção de água, sendo que a menor absorção ocorreu no tempo de 8 min e temperatura de $200^{\circ} \mathrm{C}$. Para o teor de resina de $6 \%$ a absorção de água aumenta na temperatura de $180^{\circ} \mathrm{C}$ e, em seguida, diminui novamente na temperatura de $200^{\circ} \mathrm{C}$ e a menor absorção ocorreu no tempo de 8 min e temperatura de $160^{\circ} \mathrm{C}$, para absorção 2 horas, e temperatura de 
$200^{\circ} \mathrm{C}$, para absorção 24 horas.

A

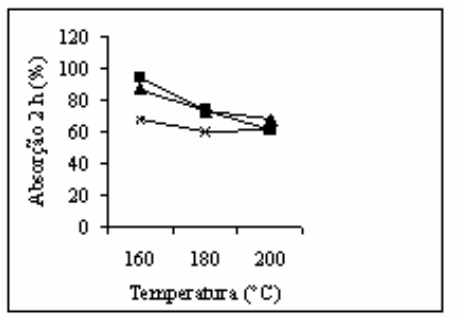

B

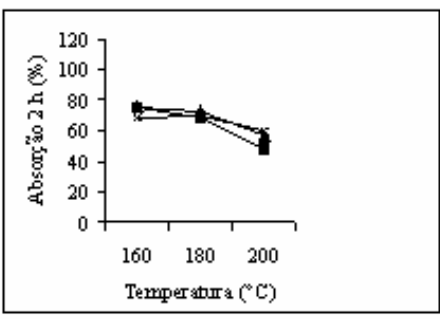

$\mathrm{C}$

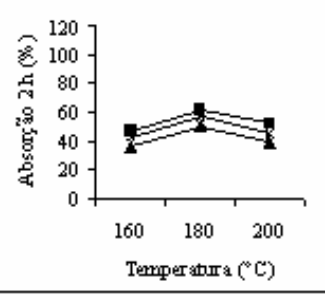

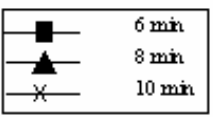

FIGURA 3: Cortes nas superfícies de resposta ajustada para a variável dependente absorção de água $2 \mathrm{~h}$, para os teores de resina 3\% (A), 4,5\% (B) e $6 \%(\mathrm{C})$.

FIGURE 3: Cuts in the surfaces of reply adjusted for the dependent variable water absorption $2 \mathrm{~h}$, based on resin content $3 \%(\mathrm{~A}), 4,5 \%(\mathrm{~B})$ and $6 \%(\mathrm{C})$.

A

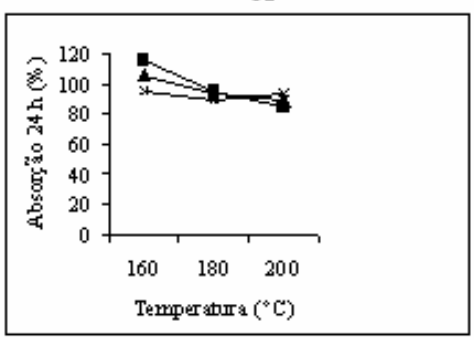

B

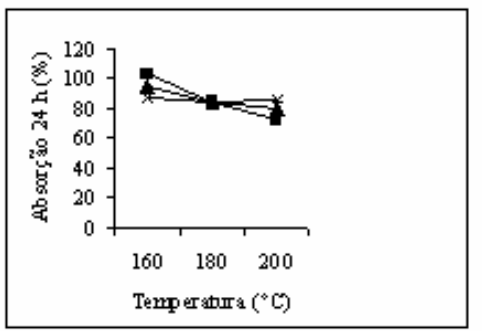

c
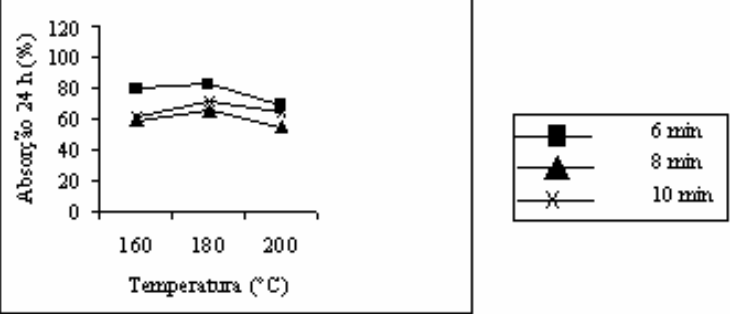

FIGURA 4: Cortes nas superfícies de resposta ajustada para a variável dependente absorção de água $24 \mathrm{~h}$ para os teores de resina 3\% (A), 4,5\% (B) e $6 \%$ (C).

FIGURE 4: Cuts in the surfaces of reply adjusted for the dependent variable water absorption $24 \mathrm{~h}$ based on resin content $3 \%(\mathrm{~A}), 4,5 \%(\mathrm{~B})$ and $6 \%(\mathrm{C})$.

A Figura 5 ilustra a tendência de comportamento da TNRE, em função dos teores de resina. De acordo com essa figura, pode-se observar que nos teores de resina de 3 e $4,5 \%$, à medida que aumenta a temperatura de prensagem diminui a TNRE. A menor TNRE ocorreu na temperatura de $2000^{\circ} \mathrm{C}$ e tempo de 6 min. Para o teor de resina de $6 \%$, à medida que aumenta a temperatura de prensagem, a TNRE se mantém constante, sendo que a menor TNRE ocorreu na temperatura de $1600^{\circ} \mathrm{C}$ e tempo de $10 \mathrm{~min}$. Esta propriedade está associada ao inchamento em espessura, em que também foi observada a mesma tendência. 
A

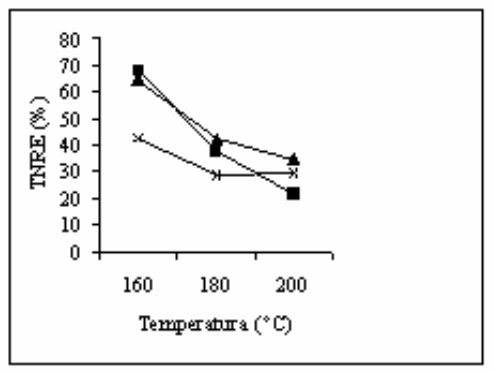

B

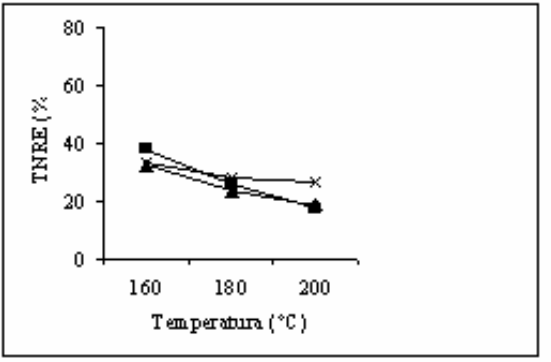

$\mathrm{C}$
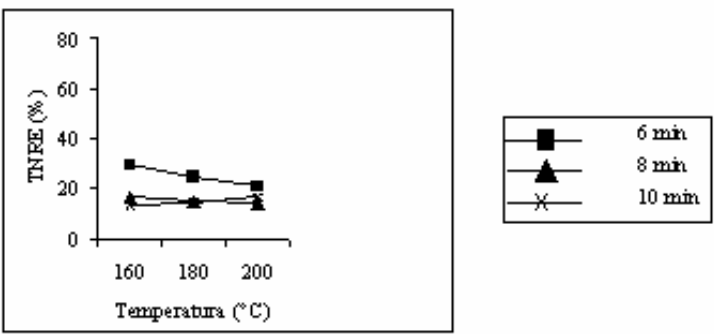

FIGURA 5: Cortes nas superfícies de resposta ajustada para a variável dependente TNRE para os teores de resina 3\%(A), 4,5\% (B) e 6\% (C).

FIGURE 5: Cuts in the surfaces of reply adjusted for the dependent variable TNRE for resin content $3 \%$ (A), $4,5 \%(\mathrm{~B})$ and $6 \%(\mathrm{C})$.

A Tabela 5 apresenta os respectivos pontos estacionários (S), para cada equação de regressão ajustada, para cada variável e sua classificação. Pode-se notar que, para todas as variáveis estudadas, a maioria desses pontos se encontra fora da área experimental (intervalo estudado) e, quando dentro, bem próximo da fronteira. Esse fato ocorre, com freqüência, em estudos de superfície de resposta, sobretudo, quando se desconhece exatamente o comportamento do fenômeno. Apesar da classificação de ponto de máximo, mínimo ou sela, segundo Meyrs (1971), o ponto não é unicamente um máximo, mínimo ou sela. O ponto se encontra numa região formada por uma aresta estacionária.

TABELA 5: Coordenadas do ponto estacionário e sua classificação.

TABLE 5: Stationary point coordinations and their classification.

\begin{tabular}{lcccc}
\hline Variável & Resina $(\%)$ & Ponto Estacionário & Classificação \\
\hline Inchamento $2 \mathrm{~h}$ & 3 & $\mathrm{X}_{1}=201,8251 ; \mathrm{X}_{2}=8,2333$ & Sela \\
& 4,5 & $\mathrm{X}_{1}=-67,7659 ; \mathrm{X}_{2}=3,4647$ & Sela \\
& 6 & $\mathrm{X}_{1}=188,5273 ; \mathrm{X}_{2}=8,9780$ & Sela \\
\hline Inchamento $24 \mathrm{~h}$ & 3 & $\mathrm{X}_{1}=203,0705 ; \mathrm{X}_{2}=8,3860$ & Sela \\
& 4,5 & $\mathrm{X}_{1}=-262,2893 ; \mathrm{X}_{2}=27,6139$ & Sela \\
& 6 & $\mathrm{X}_{1}=386,3011 ; \mathrm{X}_{2}=3,0907$ & Sela \\
\hline Absorção 2h & 3 & $\mathrm{X}_{1}=200,5816 ; \mathrm{X}_{2}=8,0493$ & Sela \\
& 4,5 & $\mathrm{X}_{1}=162,3453 ; \mathrm{X}_{2}=7,1872$ & Máximo \\
& 6 & $\mathrm{X}_{1}=181,4156 ; \mathrm{X}_{2}=8,3204$ & Sela \\
\hline Absorção & 3 & $\mathrm{X}_{1}=187,9319 ; \mathrm{X}_{2}=9,1917$ & Máximo \\
& 4,5 & $\mathrm{X}_{1}=181,6656 ; \mathrm{X}_{2}=9,9276$ & Sela \\
& 6 & $\mathrm{X}_{1}=178,6269 ; \mathrm{X}_{2}=8,5020$ & Sela \\
\hline TNRE & 3 & $\mathrm{X}_{1}=197,9006 ; \mathrm{X}_{2}=8,3262$ & Sela \\
& 4,5 & $\mathrm{X}_{1}=254,1789 ; \mathrm{X}_{2}=3,9234$ & Mínimo \\
& 6 & $\mathrm{X}_{1}=233,3872 ; \mathrm{X}_{2}=7,4115$ & Sela \\
\hline
\end{tabular}

Em que: $\mathrm{X}_{1}=$ Temperatura de prensagem $\left({ }^{\circ} \mathrm{C}\right) ; \mathrm{X}_{2}=$ Tempo de prensagem $(\mathrm{min}) ;$ TNRE $=$ Taxa de não-retorno em espessura. 


\section{CONCLUSÕES}

O teor de resina, a temperatura e o tempo de prensagem afetam, de forma significativa, a retenção de água pelos painéis.

O teste de identidade de modelos foi significativo, para todas as variáveis avaliadas no processo. Desta forma, não se pode substituir as equações, por nível de resina, por uma equação comum.

O ajuste das equações, em cada nível de resina, foi elevado para todas as variáveis.

A maioria dos pontos estacionários está fora da área experimental, indicando a necessidade de ajuste de novos modelos.

\section{REFERÊNCIAS BIBLIOGRÁFICAS}

AMERICAN SOCIETY FOR TESTING MATERIALS. Standard methods of evaluating the properties of woodbase fiber and particle materials : ASTM D-1037-91. Philladelphia: ASTM, 1998. (Annual Book of ASTM standards, vol. 04.09).

BRITO, E. O. Produção de chapas de partículas de madeira a partir de maravalhas de Pinus elliotti Engelm. Var. elliotti plantado no sul do Brasil. 1995. 123p. Dissertação (Doutorado em Engenharia Florestal) - Universidade Federal do Paraná, Curitiba, 1995.

CLOUTIER, A. Oriented stranboard (OSB): raw material, manufacturing process, properties of wood-base fiber and particle materials. In.: INTERNATIONAL SEMINAR ON SOLID WOOD PRODUCTS OF HIGH TECHNOLOGY, 1., 1998, Belo Horizonte. Anais... Belo Horizonte: SIF, 1998. p. 173-185.

IWAKIRI, S. A influência de variáveis de processamento sobre propriedades de chapas de partículas de diferentes espécies de Pinus. 1989. 129p. Dissertação (Doutorado em Engenharia Florestal) - Universidade Federal do Paraná, Curitiba, 1989.

MALONEY, T.M. Modern particleboard e dry-process fiberboard manufacturing. 2. ed. São Francisco : M. Freeman, 1993. 689 p.

MATOS, J.L.M. Ciclo de prensa em chapas de partículas estruturais "Waferboards". 1988 163p. Dissertação (Mestrado em Engenharia Florestal) - Universidade Federal do Paraná, Curitiba, 1988.

MENDES, L.M. Pinus spp. na produção de painéis de partículas orientadas (OSB). 2001. 156p. Dissertação (Doutorado em Engenharia Florestal) - Universidade Federal do Paraná, Curitiba, 2001.

MENDES, L.M.; IWAKIRI, S. Perspectivas para o uso da madeira de eucalipto para produção de OSB (Oriented Strand Board). In: SEMINÁRIO SÓLIDOS DE EUCALIPTO: avanços científicos e tecnológicos, 1., 2002, Lavras. Anais... Lavras, 2002. p. 156-165.

MEYRS, R.N. Response surface methodology. Boston : Allyn and Bacon, 1971. 246 p. 